\title{
Return of the 'spacebugs'
}

DOI:

10.1038/nrmicro1782
The detrimental effect on the human immune system of a low-gravity environment such as that experienced during space flight has been well established, but the effects on bacteria that infect humans has not been probed. New findings published in the Proceedings of the National Academy of Sciences USA show that the space-flight environment affects the cellular and physiological responses of Salmonella enterica serovar Typhimurium (S. typhimurium) and, surprisingly, increases the virulence of this strain.

Wilson, Nickerson and colleagues tested the responses of a virulent $S$. typhimurium strain during a flight of the Space Shuttle Atlantis. Cultures were activated during the space mission and grown for a specified time period, and were fixed, using an RNA or protein fixative, to terminate growth. All samples were compared with identical cultures that were grown simultaneously on the ground at the Kennedy Space Center. The transcriptome and proteome were examined to evaluate how space flight affected gene and protein expression. Exposure to space-flight conditions caused the differential expression of 167 genes and 73 proteins compared with groundcontrol cultures. The RNA-binding protein Hfq was identified as a global regulator that is involved with aspects of the tested responses. Hfq has a documented role in stress responses and the virulence of many bacterial pathogens, including S. typhimurium.

The virulence of space-flight or synchronous ground-control strains was assessed by infecting BALB/c mice. Space-flight cultured S. typhimurium killed more mice and had a decreased $\mathrm{LD}_{50}$ value and decreased time to death compared with control strains. These intriguing results indicate that strains that are exposed to space-flight conditions (low gravity) display increased virulence compared with strains grown under normal-gravity conditions.

The authors propose that a signal is sensed during exposure to lowgravity conditions which induces global alterations that, ultimately, result in increased virulence. This fascinating study has important implications for the assessment of disease risk during space flight and the production of novel antibacterial therapeutics that target Hfq.

Gillian Young

ORIGINAL RESEARCH PAPER Wilson, J. W. et al. Space flight alters bacterial gene expression and virulence and reveals a role for global regulator Hfq. Proc. Natl Acad. Sci. USA 6, 144-152 (2007)



Astronaut Heide Stefanyshyn-Piper activating the growth of bacteria in a specially sealed growth chamber during mission STS-115 on board the Space Shuttle Atlantis in 2006. Photograph kindly provided by NASA (National Aeronautics and Space Administration). 